\title{
ENERGY DEPOSITION STUDIES OF BLOCK-COIL QUADRUPOLES FOR THE LHC LUMINOSITY UPGRADE*
}

\author{
N.V. Mokhov\#, V.V. Kashikhin, M.E. Monville, Fermilab, IL 60510, U.S.A. \\ P. Ferracin, G.L. Sabbi, LBNL, Berkeley, CA 94720, U.S.A.
}

\begin{abstract}
At the LHC upgrade luminosity of $10^{35} \mathrm{~cm}^{-2} \mathrm{~s}^{-1}$, collision product power in excess of a $\mathrm{kW}$ is deposited in the inner triplet quadrupoles. The quadrupole field sweeps secondary particles from pp-collisions into the superconducting (SC) coils, concentrating the power deposition at the magnetic mid-planes. The local peak power density can substantially exceed the conductor quench limits and reduce component lifetime. Under these conditions, block-coil geometries may result in overall improved performance by removing the superconductor from the magnetic mid-planes and/or allowing increased shielding at such locations. First realistic energy deposition simulations are performed for an interaction region based on block-coil quadrupoles with parameters suitable for the LHC upgrade. Results are presented on distributions of power density and accumulated dose in the inner triplet components as well as on dynamic heat loads on the cryogenic system. Optimization studies are performed on configuration and parameters of the beam pipe, cold bore and cooling channels. The feasibility of the proposed design is discussed.
\end{abstract}

\section{INTRODUCTION}

Several possible upgrade paths are under consideration to achieve a luminosity capability of $10^{35} \mathrm{~cm}^{-2} \mathrm{~s}^{-1}$ in the LHC Interaction Regions (IR) IP1 and IP5 [1, 2]. Recent progress in the development of $\mathrm{Nb}_{3} \mathrm{Sn}$ superconductor enables one to consider $\mathrm{Nb}_{3} \mathrm{Sn}$ magnets as possible second generation quadrupoles for the LHC IR. The quadrupole fields sweep the secondary particles from pp-collisions at the Interaction Point (IP) into the SC coils along the vertical and horizontal planes, giving rise to a local peak power density $\varepsilon_{\max }$ that can substantially exceed the quench limits at such luminosity, with drastically reduced component lifetime and $\mathrm{kW}$-level radiation loads on the inner triplet cryogenic system [3]. Only with a sophisticated highly-efficient protection system implemented into an inner triplet $[3,4]$ can one consider realistic upgrade scenarios. A novel quadrupole design, based on $\mathrm{Nb}_{3} \mathrm{Sn}$ block-type coils, has a potential of mitigating the problems described.

\section{BLOCK-TYPE COIL QUADRUPOLES}

The block-type quadrupole considered for this energy deposition study is depicted in Fig. 1. The design consists of two double-layer coils of the flat racetrack type. The

\footnotetext{
*Work supported by Fermi Research Alliance, LLC, under contract No. DE-AC02-07CH11359 with the U.S. Department of Energy. \#mokhov@ fnal.gov

This work was supported by the US DOE Contract DE-AC0205CH11231.
}

coils are placed around a square aperture and rotated of $45^{\circ}$ with respect to the horizontal axis. Spacers are included on the mid-plane to provide vertical support to the conductor blocks. The coils utilize a flat cable with the same main parameters of the one implemented in the LARP TQ quadrupole series [5]: 27 strands of $0.7 \mathrm{~mm}$ diameter, $10.05 \mathrm{~mm}$ width, 1.260 thickness, and 0.125 $\mathrm{mm}$ thick insulation. The coil cross-section features a half-aperture of $55 \mathrm{~mm}$ on the mid-plane and 5 conductor blocks whose positions have been optimized to minimize the geometric harmonics (less than 0.1 units at a reference radius of $22 \mathrm{~mm}$ ). Assuming a current density of 3000 $\mathrm{A} / \mathrm{mm}^{2}$ at $12 \mathrm{~T}, 4.2 \mathrm{~K}$, the quadrupole generates a maximum (short sample) gradient of $255 \mathrm{~T} / \mathrm{m}$ at $1.9 \mathrm{~K}$, with a peak field in the conductor of $14.2 \mathrm{~T}$.

The support structure (Fig. 1, right) is designed to provide pre-stress to the coil and withstand the Lorentz forces during magnet excitation. The coils are supported by four bolted stainless steel pads surrounded by a fourpiece iron yoke and an aluminum shell. The assembly is done by means of the key and bladder technology [5], which allows pre-tensioning the shell at room temperature, providing at the same time an initial precompression to the coil pack. During cool-down, the different thermal contraction between aluminum and iron further increases the coil pre-stress, so that no separation between conductors and winding poles is expected when Lorentz forces are applied.

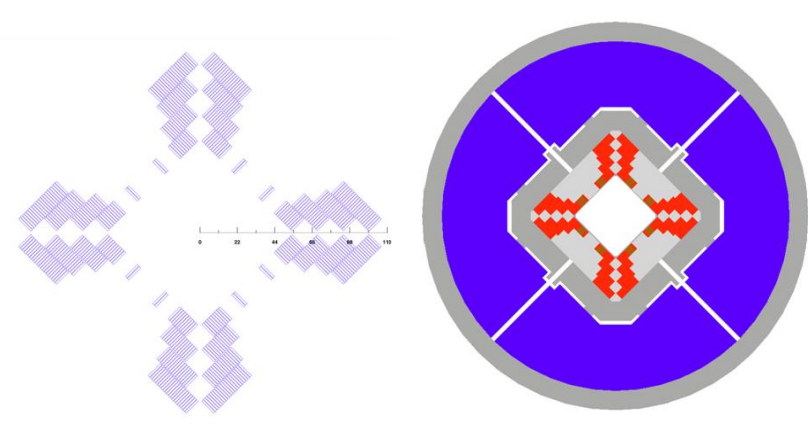

Figure 1: Coil (left) and magnet (right) cross-section.

\section{MARS15 MODELING}

All the energy deposition calculations in this study are performed with the current version of the MARS15 code [6]. The LHC IP5 inner triplet layout adjusted to a luminosity of $10^{35} \mathrm{~cm}^{-2} \mathrm{~s}^{-1}$ along with the TAS front absorber, beam pipes and other near-beam components are used in the MARS model (Fig. 2). The half-crossing angle at the IP is 0.212 mrad. The block-type coil quadrupole geometry, materials and OPERA-calculated 
magnetic field maps are implemented into the MARS model and thoroughly tested. Note that the model includes also four MCBX and MQSX corrector magnets as shown in Fig. 2.

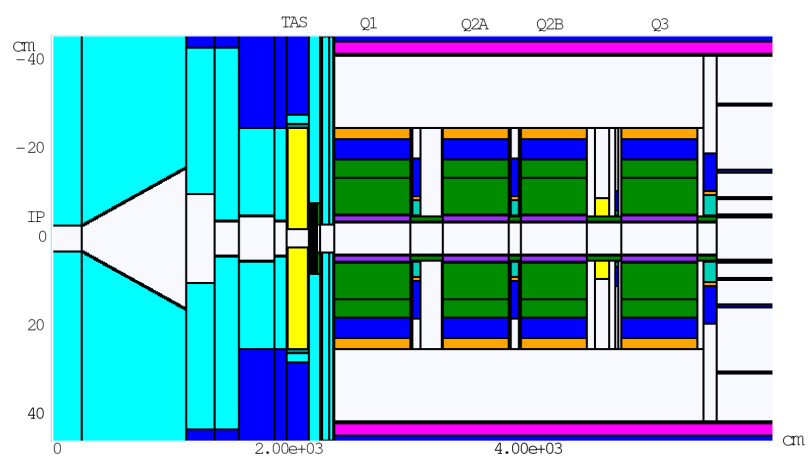

Figure 2: LHC IP5 MARS15 model.

In this study, all the design constraints related to the energy deposition are obeyed [3]: quench stability, radiation damage, dynamic heat loads, hands-on maintenance and corresponding engineering constraints. The quench limit accepted for the $\mathrm{Nb}_{3} \mathrm{Sn}$ IR magnets is 5 $\mathrm{mW} / \mathrm{g}$, that - with a required safety margin of a factor of 3 - gives the design goal for the peak power density averaged over the $\mathrm{SC}$ block $\varepsilon_{\max }=1.7 \mathrm{~mW} / \mathrm{g}$.

The main advantage of a block-coil geometry - from an energy deposition standpoint, i.e. the absence of the superconductor in the magnetic mid-planes and the space available in the same locations for shielding, has given us a possibility to find a configuration shown in Fig. 3 which is compatible with the design goals.

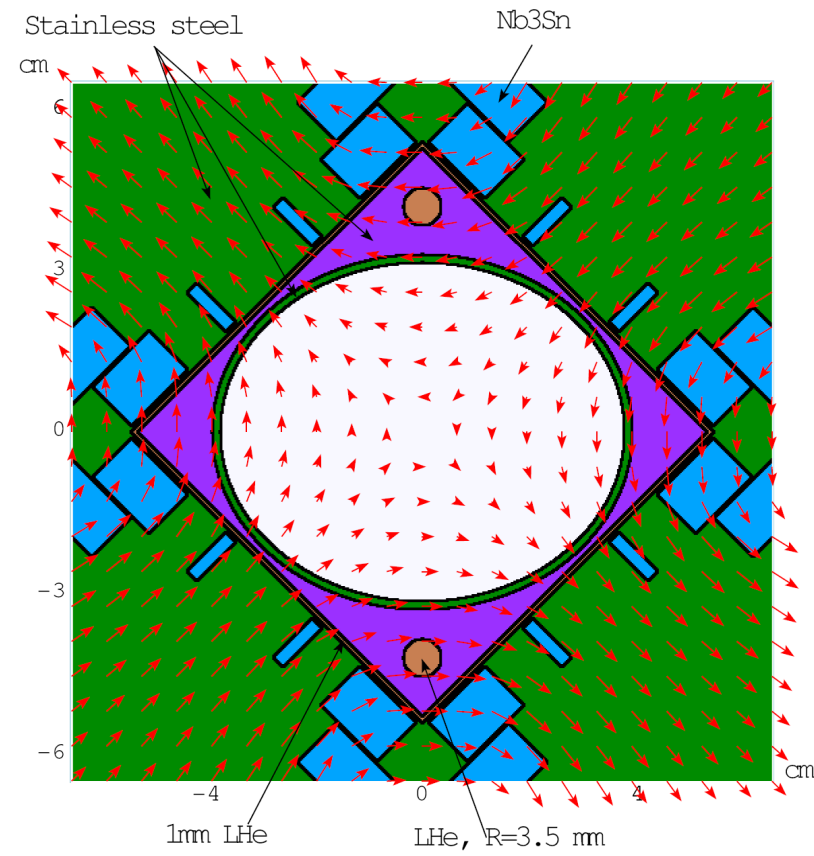

Figure 3: Quadrupole coil, absorber and cooling channel geometry as in MARS15.

\section{RESULTS}

Fig. 4 shows power density profiles in the hottest SC coils along the inner triplet magnets. The distribution peaks at the non-IP end of the first quadrupole. A transverse profile at this location is given in Fig. 5. One can see how substantial is the reduction of energy deposition in SC coils achieved with the absorber proposed.

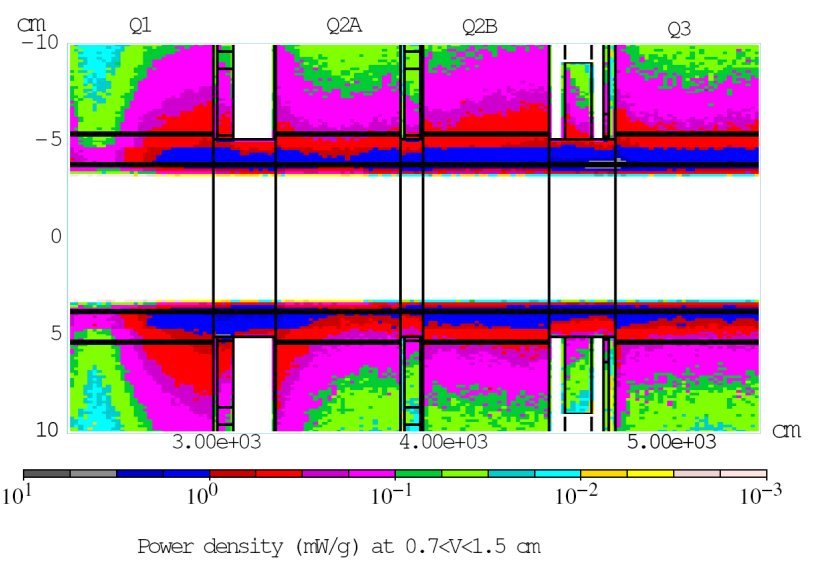

Figure 4: Longitudinal profiles of power density in the block coils closest to the mid-plane.

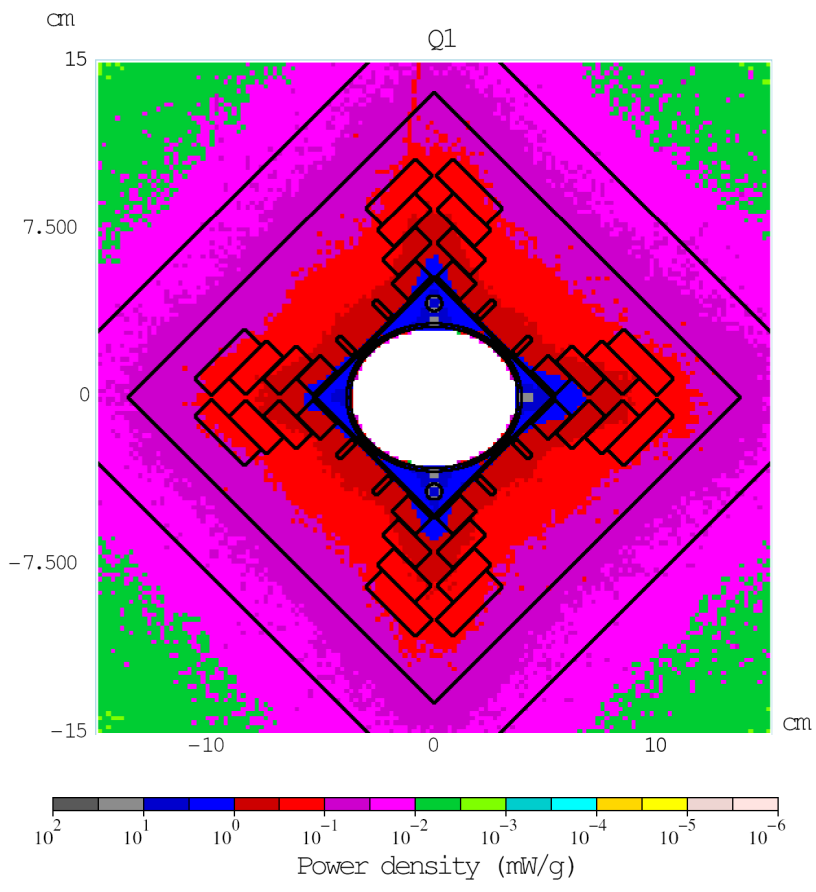

Figure 5: Power density isocontours at the Q1 non-IP end.

Maximum power densities averaged over the hottest SC block coils at the longitudinal peaks in the quadrupoles and in four corrector magnets are presented in Fig. 6. One can see that for the block-type coil design and the absorber proposed, even the maximum value of $0.9 \mathrm{~mW} / \mathrm{g}$ at the non-IP end of the first quadrupole is lower than the design goal of $\varepsilon_{\max }=1.7 \mathrm{~mW} / \mathrm{g}$ at the luminosity of $10^{35}$ $\mathrm{cm}^{-2} \mathrm{~s}^{-1}$. 


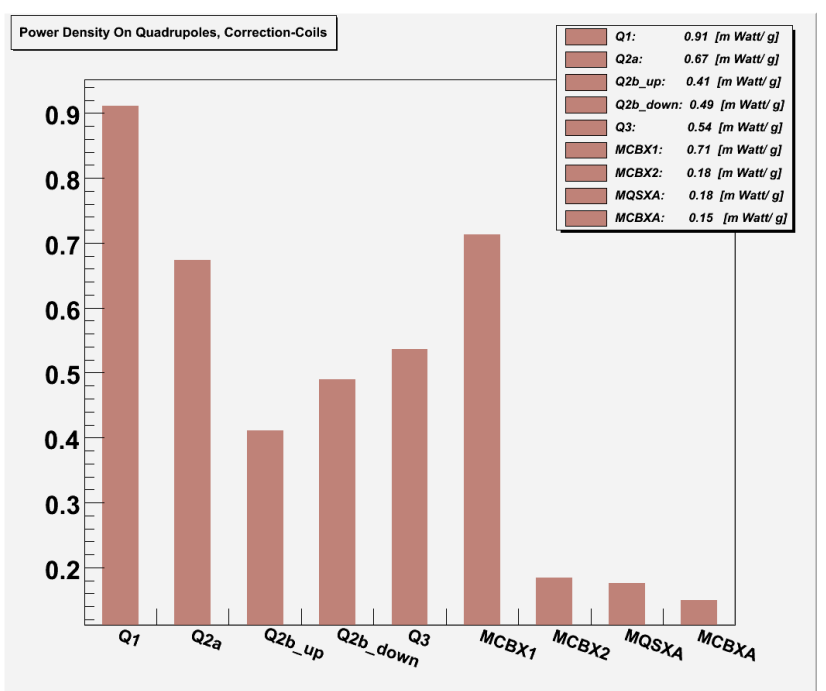

Figure 6: Peak power density in the SC coils of the inner triplet quadrupoles and corrector magnets.

Luminosity driven dynamic heat loads on quadrupoles and corrector magnets - total and SC coils only - are shown in Fig. 7. Not a surprise, a 10-fold increase of total loads is expected at ten times higher luminosity compared to the nominal case [3]. About $0.3 \mathrm{~kW}$ of power is dissipated in each of the quadrupole, with loads on the SC coils at a $0.04 \mathrm{~kW}$ level. The total heat to be removed by the cryogenic system is $1.36 \mathrm{~kW}$ on either side of the high-luminosity IRs. This is a quite challenging problem.

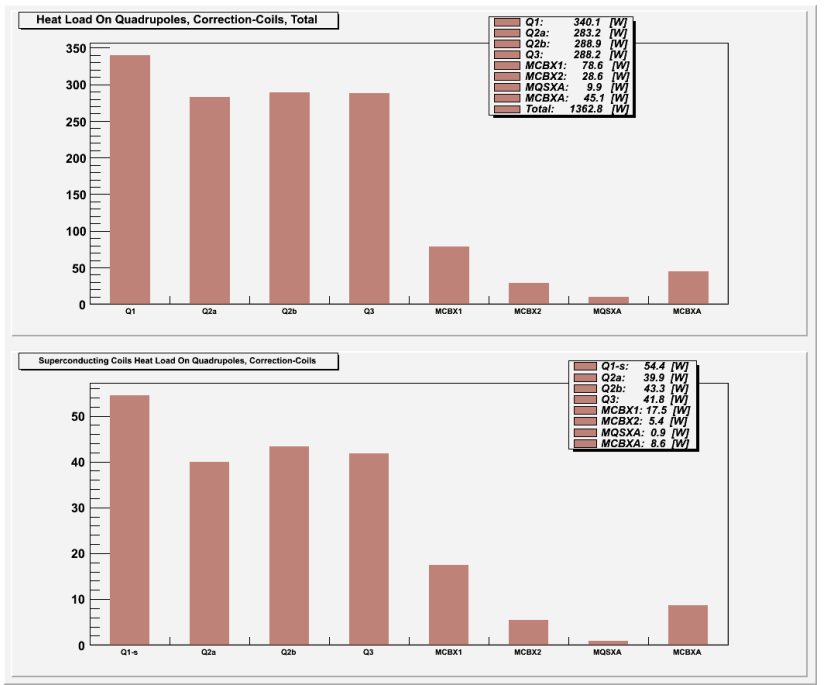

Figure 7: Dynamic heat loads on quadrupoles and correctors: total (top) and SC coils only (bottom).

Dose absorbed in the hottest spots of the SC coils defines the life-time of the magnets. Both longitudinal and transverse dose profiles are similar to those for power density shown in Figs. 4-5. Assuming $10^{7} \mathrm{~s}$ of physics data taking per year at the luminosity of $10^{35} \mathrm{~cm}^{-2} \mathrm{~s}^{-1}$, one gets the maximum doses in SC coils of up to $50 \mathrm{MGy} / \mathrm{yr}$ as shown in Fig. 8.

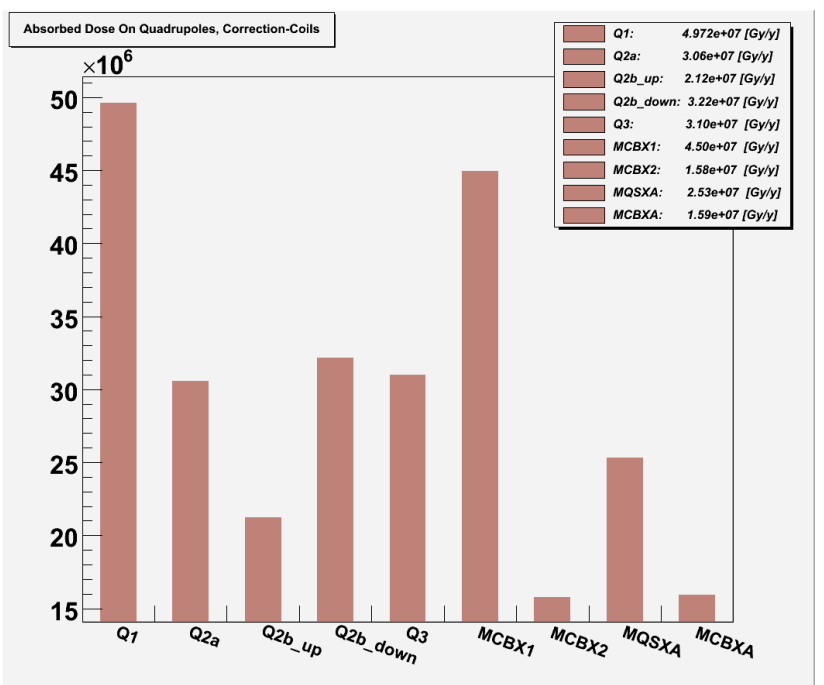

Figure 8: Peak absorbed dose in the SC coils of the inner triplet quadrupoles and corrector magnets.

Residual dose on the beam pipes, absorbers and other near-beam parts in the region scales roughly with the luminosity, i.e., ten times higher compared to the nominal luminosity case, exceeding $1000 \mathrm{mSv} / \mathrm{hr}$ at hot locations. At the same time, the dose on the outer vessel is only slightly higher than for the nominal case because of the larger amount of absorbing material.

\section{SUMMARY}

The LHC inner triplet based on the block-coil quadrupoles with the proposed configuration of absorbers and cooling is feasible from the energy deposition standpoint and compatible with the LHC upgrade luminosity of $10^{35} \mathrm{~cm}^{-2} \mathrm{~s}^{-1}$.

\section{REFERENCES}

[1] J.B. Strait, et al., “Towards a New LHC Interaction Region Design for a Luminosity Upgarde", PAC2003 Proc., p. 42; Fermilab-Conf-03/098 (2003), LHC Project Report 643 (2003).

[2] J.B. Strait, N.V. Mokhov, T. Sen, "Overview of Possible LHC IR Upgrade Layouts", Fermilab-Conf05/007-AD-E-T (2005).

[3] N.V. Mokhov et al., "Protecting LHC IP1/IP5 Components Against Radiation Resulting from Colliding Beam Interactions", Fermilab-FN-732 (2003); LHC Project Report 633, CERN (2003).

[4] N.V. Mokhov, I.L. Rakhno, "Mitigating Radiation Loads in Nb3Sn Quadrupoles for the CERN Large Hadron Collider Upgrades", Phys. Rev. Special Topics - Accelerators and Beams, 9, 101001 (2006).

[5] S. Caspi et al., IEEE Trans. Appl. Superconduct., Vol. 16, no. 2, June 2006, p. 358.

[6] N.V. Mokhov, "The MARS Code System User's Guide", Fermilab-628 (1995); Fermilab-Conf-04/053 (2004); http://www-ap.fnal.gov/MARS/. 